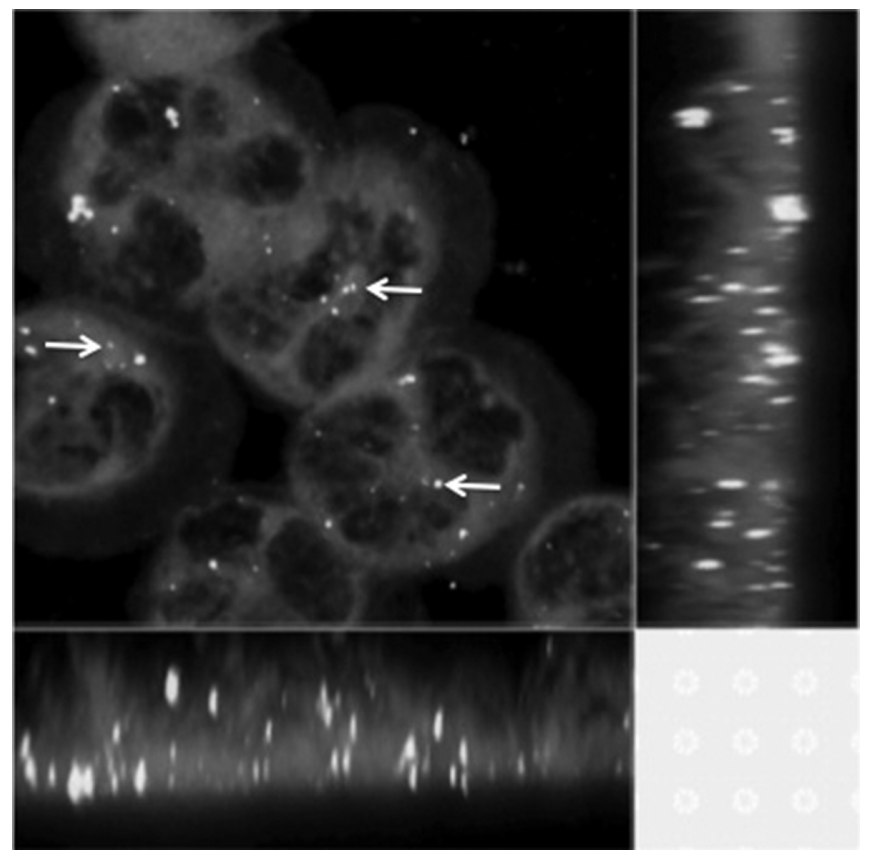

Abstract S87 Figure 1 Indirect fluorescent confocal microscopy was carried out on cytospins of neutrophils purified from adult blood and incubated with RSV for a $2 \mathrm{~h}$ in the presence of GM-CSF. Z stacks were taken using a Leica confocal and Z-projection images produced using Imaris software. RSV (white arrow) is identified in all depths of the cytoplasm in this orthogonal view throughout the cell in discrete pockets suggestive of endosomes

Introduction and objectives Respiratory Syncytial Virus (RSV) is a major cause of lower respiratory tract infection during infancy. Neutrophils are the predominant cell type within the RSVinfected airway, $80 \%$ of inflammatory cells from BAL of intubated infants being neutrophils. Despite extensive research unpicking innate viral responses to RSV little is known about the specific role the neutrophil plays. The aim of this work was to investigate the response of neutrophils to RSV using an in vitro model utilising neutrophils from adult volunteers. To investigate whether relative immaturity of infant neutrophils leads to impaired responses we are now comparing adult neutrophils with neonatal neutrophils, isolated from cord blood.

Methods Highly purified neutrophils from whole blood of healthy adult donors were incubated with RSV. Samples were taken at 2, 4, and $20 \mathrm{~h}$ for QT-PCR of RSV $\mathrm{N}$ gene, western blot analysis and cytospin slides for confocal imaging of RSV F protein. Experiments were then replicated using neutrophils purified from cord blood, collected from the placenta following elective caesarean section, of healthy term neonates. Supernatants were stored for measurement of the cytokine response of the neutrophil to RSV.

Results Uptake of RSV by adult neutrophils was shown by both western blot and quantitative RT-PCR. Maximal uptake was at 4 $\mathrm{h}$ with a reduction by $20 \mathrm{~h}$. Confocal microscopy was undertaken, using a primary monoclonal antibody to RSV fusion protein. This showed that RSV was internalised inside the cytoplasm in a distribution suggestive of endosomal uptake (see figure). Preliminary data suggests that neonatal neutrophils are also capable of this viral uptake but work is ongoing to determine differences between the two models.

Conclusions We have shown that neutrophils may be involved in viral clearance as part of the immune response to viral invasion. They appear to take up virus with kinetics suggestive of endocytosis. Work is ongoing to establish the mechanism of entry using a panel of inhibitors. Initial results would suggest that neonatal neutrophils may respond similarly but work continues to establish whether this is the case and if there is any functional impairment that may explain infants' propensity to severe disease.

\section{S88 ELECTRON TOMOGRAPHY DETECTS ULTRASTRUCTURAL ABNORMALITIES IN PATIENTS WITH PCD DUE TO A DNAH11 DEFECT}

${ }^{1} \mathrm{R}$ Kwan, ${ }^{2} \mathrm{~T}$ Burgoyne, ${ }^{3} \mathrm{M}$ Dixon, ${ }^{2} \mathrm{M}$ Patel, ${ }^{2} \mathrm{~J}$ Scully, ${ }^{2} \mathrm{~A}$ Onoufriadis, ${ }^{3} \mathrm{C}$ Hogg, ${ }^{2} \mathrm{HM}$ Mitchison, ${ }^{3} \mathrm{~A}$ Shoemark. ${ }^{1}$ Imperial College, London, UK; ${ }^{2}$ University College London, London, UK; ${ }^{3}$ Royal Brompton Hospital, London, UK

\subsection{6/thoraxjnl-2014-206260.94}

Primary Ciliary Dyskinesia (PCD) is a genetically heterogeneous condition. characterised by impaired mucociliary clearance and chronic sino-pulmonary disease. Over 30 causative gene mutations resulting in dysfunctional ciliary motility and structure have been identified. Ciliary ultrastructure examined by Transmission Electron Microscopy (TEM) is currently the diagnostic gold standard. Patients with mutations in the gene DNAH11 have a hyperfrequent ciliary beat and clinical symptoms of PCD. Diagnosis of a DNAH11 defect by TEM is difficult due to apparently normal ciliary ultrastructure. The electron tomography technique, an extension of TEM, produces 3D models of cilia with superior resolution.

The aim of this study was to determine if electron tomography can detect ultrastructural abnormalities in patients with DNAH11 defects.

Immunofluorescence by specific antibodies for DNAH11 showed localisation to the proximal portion of cilia. Proximal cilia cross sections from araldite embedded nasal brush biopsies were examined. Dual axis tomograms were collected on a Philips CM200 electron microscope. The data were analysed using IMOD software and averaged using PEET.

Electron tomography and averaging of cilia cross sections indicated a deficiency in the outer dynein arm, consistent across

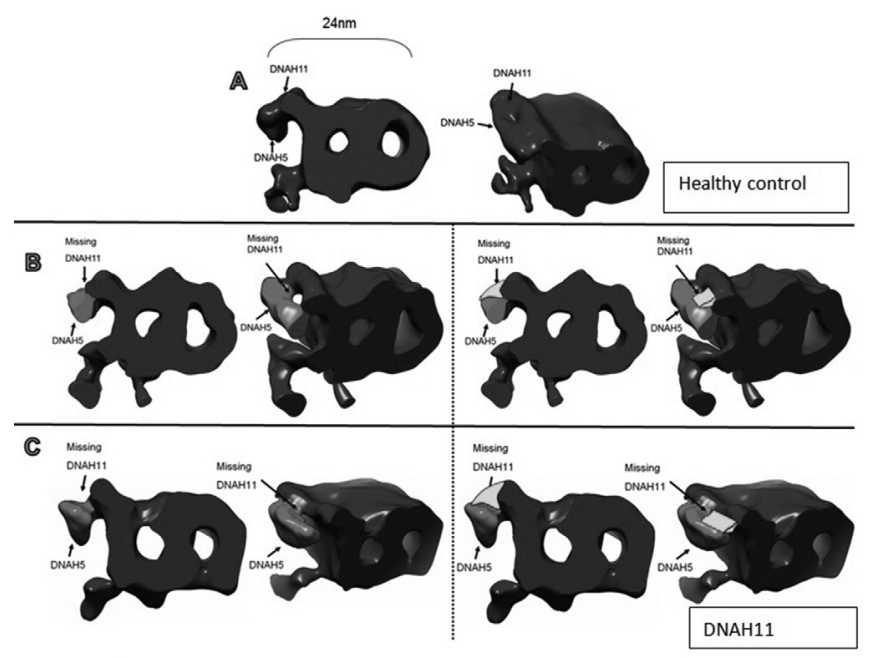

Abstract S88 Figure 1 Averaged microtubule doublets imaged using Chimaera. (A) normal control (B) and (C) DNAH11 mutations with DNAH5 highlighted in red, showing the missing DNAH11 (left two images) and the proposed DNAH11 shapes inserted, coloured green (right two images) 
all patients with a DNAH11 defect $(\mathrm{n}=5)$ compared to healthy controls $(n=3)$ and patients with PCD due to a defect of the central pair or nexin link $(\mathrm{n}=3)$ (Figure 1). A reduction in outer dynein arm volume of $30 \%$ was identified compared to healthy and PCD controls.

In conclusion, a mutation in DNAH11 results in a subtle abnormality in ultrastructure. The defects are specific to the 'forearm' of the outer dynein arm and only detectable at the base of the cilium where DNAH11 is located. Electron tomography is highly effective in visualising this defect.

\section{S89 IDENTIFICATION OF POTENTIALLY PATHOGENIC MICROORGANISMS BY SELECTED ION FLOW TUBE - MASS SPECTROMETRY (SIFT-MS)}

${ }^{1} \mathrm{JM}$ Jackson, ${ }^{2} \mathrm{VL}$ Kim, ${ }^{3} \mathrm{AC}$ Tuck, ${ }^{1} \mathrm{SA}$ Wootton, ${ }^{4} \mathrm{TM}$ Wilkinson. ${ }^{1} \mathrm{NIHR}$ Southampton Biomedical Research Centre, Southampton, UK; ${ }^{2}$ NIHR Southampton Respiratory Biomedical Research Unit, Southampton, UK; ${ }^{3}$ Infectious Disease Epidemiology Group, Sir Henry Wellcome Laboratories, Academic Unit of Clinical and experimental Sciences, Faculty of Medicine, University of Southampton, Southampton, UK; ${ }^{4}$ University Hospital Southampton NHS Foundation Trust, Southampton, UK

\subsection{6/thoraxjn-2014-206260.95}

Introduction Improved targeted therapy and reduced clinical burden from respiratory bacterial infection could result from early identification of specific Potential Pathogenic Microorganisms (PPM). Sputum culture for identification usually requires several days. SIFT-MS has the potential to reduce the required incubation time by sampling the culture headspace to generate an ionic spectrum from volatile organic compounds that may be characteristic of the PPM. Additionally, these signatures may be detectable in breath taken directly from patients. This pilot study investigates the potential of SIFT-MS to identify 5PPMs, incubated separately for $24 \mathrm{hr}$.

Methods Training set: Haemophilus influenzae (HI), Moraxella catarrhalis (MC), Streptococcus pneumoniae (SP), Staphylococcus aureus (SA) and Pseudomonas aeruginosa (PA) cultures and a negative control incubated at $37^{\circ} \mathrm{C}$ on chocolate agar plates in sealed bags for $24 \mathrm{hr}, 48 \mathrm{hr}$ and $72 \mathrm{hr}$. Plates were opened for $10 \mathrm{~min}$ at room temperature before ionic spectra of the gas above the culture dishes in the range 15 to 200 mass units were recorded using SIFT-MS and standardised to operating conditions. Test set: the same five PPMs and a negative control were incubated in triplicate for $24 \mathrm{hr}$ only and analysed as above.

Results Using the spectra generated with $\mathrm{H} 3 \mathrm{O}+$ ionisation, 6 ion sets were identified. The sum of ions within each set, expressed as a percentage of the total ion sum of masses 15 to 200 (excluding reagent ions) fell into ranges that, in combination, differentiated between the PPMs. This set of conditions was incorporated into an algorithm that was then applied to the test set of triplicate plates. The algorithm correctly differentiated all $24 \mathrm{hr}$ plates with MC, SP, SA and PA from each other and from the negative control and HI plates with 100\% accuracy. Negative control and HI could not be differentiated.

Conclusion This pilot study illustrates the potential for SIFT-MS to identify monocultures of 4 common PPMs within a short incubation time and encourages further study with a wider range of pathogens alone and in combination. Early identification of PPMs in culture, and translation to potentially detect carriage or infection with specific pathogens in breath may improve management of respiratory infections.
S90 ROLE FOR IL-1ALPHA IN VIRAL-INDUCED INFLAMMATORY RESPONSES IN A CO-CULTURE MODEL OF THE AIRWAY MUCOSA

${ }^{1} \mathrm{~A}$ Hill, ${ }^{1} \mathrm{~L}$ Tezera, ${ }^{1} \mathrm{C}$ Blume, ${ }^{2} \mathrm{C}$ Grainge, ${ }^{1} \mathrm{DE}$ Davies, ${ }^{1} \mathrm{EJ}$ Swindle. ${ }^{1}$ University of Southampton (Clinical and Experimental Sciences, Faculty of Medicine), Southampton, UK; ${ }^{2}$ University of Newcastle (School of Medicine and Public Health), Newcastle, Australia

\subsection{6/thoraxjnl-2014-206260.96}

Introduction Asthma is an inflammatory disease of the conducting airways which is exacerbated by environmental exposures, such as viral infections. Bronchial epithelial cells (BECs) together with underlying fibroblasts form an epithelial mesenchymal trophic unit (EMTU) that maintains normal tissue homeostasis. In asthma the EMTU is dysregulated. Recent evidence suggests that viral infections activate the epithelial barrier resulting in mediator release which could potentially activate fibroblasts. Therefore, we hypothesised that exposure to viruses activates inflammatory and anti-viral responses in the EMTU.

Methods The EMTU was modelled using a co-culture system of polarised BECs (16HBE14o-) and fibroblasts (MRC5s) maintained on the apical and basolateral surface of a nanoporous membrane respectively. After 6 days the model was challenged apically with poly (I:C) (a viral mimetic) and barrier responses were determined by measuring transepithelial resistance (TER) while cytokine release was determined by ELISA.

Results Following poly (I:C) stimulation a significant reduction in TER was observed in both the EMTU model and BEC monocultures. However, the EMTU model maintained a higher TER at 6-24 h after challenge. With regards to cytokine secretion, poly (I:C) stimulation significantly induced pro-inflammatory (IL-6, IL-8, GM-CSF and IL-1 $\alpha$ ) and anti-viral (IP-10) mediator release from BEC but not fibroblast monocultures. In the EMTU model, basolateral IL-6, IL-8, GM-CSF and IP-10 responses to poly (I:C) were significantly enhanced compared to BEC monocultures. In addition, basolateral pro-inflammatory (IL-6, IL-8 and GM-CSF) but not antiviral (IP-10) responses to poly (I:C) were abrogated in the EMTU model after pre-incubation with IL-1 receptor antagonist (IL-1ra). Furthermore, direct stimulation with IL- $1 \alpha$ induced IL- 6 and IL- 8 release in the EMTU model and fibroblast monocultures but not in BEC monocultures.

Conclusions Poly (I:C) activates inflammatory and anti-viral responses in BEC monocultures and fibroblast co-culture models of the EMTU. These responses were enhanced in the co-culture model suggesting that the EMTU is activated. Inflammatory but not anti-viral responses were mediated by epithelial-derived IL$1 \alpha$ acting on the underlying fibroblasts. This may have important consequences in promotion of inflammation and airway remodelling in viral-induced exacerbations of asthma.

\section{New asthma treatments}

\section{S91 ONCE-DAILY TIOTROPIUM RESPIMAT® ADD-ON TO ICS + LABA IMPROVES SYMPTOM CONTROL AND REDUCES EXACERBATIONS IN PATIENTS WITH SYMPTOMATIC ASTHMA}

${ }^{1} \mathrm{D}$ Price, ${ }^{2} \mathrm{M}$ Engel, ${ }^{2} \mathrm{P}$ Moroni-Zentgraf, ${ }^{2} \mathrm{H}$ Schmidt, ${ }^{3} \mathrm{R}$ Dahl, ${ }^{4} \mathrm{P}$ Paggiaro, ${ }^{5} \mathrm{M}$ Vandewalker, ${ }^{6}$ HAM Kerstiens, ${ }^{7}$ A Kaplan. ${ }^{1}$ Institute of Applied Health Sciences, University of Aberdeen, Aberdeen, UK; ${ }^{2}$ Boehringer Ingelheim Pharma GmbH and Co. KG, Biberach an Der Riss, Germany; ${ }^{3}$ Odense University Hospital, Odense, Denmark; ${ }^{4}$ University of Pisa, Pisa, Italy; ${ }^{5}$ Clinical Research of the Ozarks, Colombia, USA; ${ }^{6}$ University Medical Center Groningen, Groningen, The Netherlands; ${ }^{7}$ Family Physician Airways Group of Canada, Ontaria, Canada

10.1136/thoraxjnl-2014-206260.97 